\title{
A Highly-collimated Water Maser Bipolar Outflow in the Cepheus A HW3d Massive Protostellar Object
}

\author{
James O. Chibueze ${ }^{1,2}$, Hiroshi Imai ${ }^{1}$, Daniel Tafoya ${ }^{1}$, \\ Toshihiro Omodaka ${ }^{1}$, Osamu Kameya ${ }^{3}$, Tomoya Hirota ${ }^{3}$, \\ Sze-Ning Chong ${ }^{1}$, and José M. Torrelles ${ }^{4}$ \\ ${ }^{1}$ Department of Physics and Astronomy, Graduate School of Science and Engineering, \\ Kagoshima University, 1-21-35 Korimoto, Kagoshima 890-0065, Japan \\ email: james@milkyway.sci.kagoshima-u.ac.jp \\ ${ }^{2}$ Department of Physics and Astronomy, Faculty of Physical Sciences, \\ University of Nigeria, Carver Building, 1 University Road, Nsukka, Nigeria \\ ${ }^{3}$ Mizusawa VLBI Observatory, National Astronomical Observatory of Japan, 2-21-1 Osawa, \\ Mitaka, Tokyo 181-8588, Japan \\ ${ }^{4}$ Instituto de Ciencias del Espacio (CSIC)-UB/IEEC, Facultat de Física, \\ Universitat de Barcelona, Martí i Franquès 1, E-08028 Barcelona, Spain
}

\begin{abstract}
We report the results of multi-epoch very long baseline interferometry (VLBI) water $\left(\mathrm{H}_{2} \mathrm{O}\right)$ maser observations carried out with the VLBI Exploration of Radio Astrometry (VERA) toward the HW3d object within the Cepheus A star-forming region. We measured proper motions of 30 water maser features, tracing a compact bipolar outflow. This outflow is highly collimated, extending through $\sim 400$ mas (290 AU), and having a typical proper motion velocity

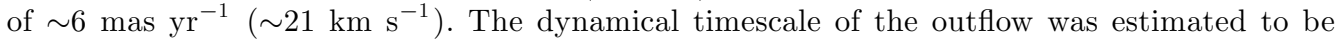
$\sim 100$ years, showing that the outflow is tracing a very early star-formation phase. Our results provide strong support that the HW3d object harbors an internal massive protostar, as previous observations suggested. In addition, we have analyzed Very Large Array (VLA) archive $1.3 \mathrm{~cm}$ continuum data of the 1995 and 2006 epochs obtained towards Cepheus A. These results indicate possible different protostars around HW3d and/or strong variability in its radio continuum emission.
\end{abstract}

Keywords. ISM: individual objects (Cepheus A), ISM: jets and outflows, masers: $\mathrm{H}_{2} \mathrm{O}$, stars: formation

\section{Introduction}

Complex environments due to the close proximity of many young massive stellar objects characterize the formation of massive stars. Sites of massive star formation are usually large distances away from the Earth ( few kpc), and their relatively short formation timescale of $\sim 10^{5}$ yr underscores the difficulty in understanding their formation and evolution (Zinnecker \& Yorke 2007). There are a number of competing concepts on how high-mass stars form. Among them are the concept of low (or medium) mass star mergers proposed by Bonnell et al. (1998); competitive accretion in protostellar cluster environment proposed by Bonnell \& Bate (2006); and the concept of gravitational collapse/high core accretion rate (Yorke \& Sonnhalter 2002).

Previous results of water $\left(\mathrm{H}_{2} \mathrm{O}\right)$ maser studies in high-mass star-forming regions show that $\mathrm{H}_{2} \mathrm{O}$ maser spatio-kinematics can trace star-formation activities at different evolutionary phases. Cepheus A (Cep A) at a distance of 700 pc (Dzib et al. 2011) is one of the closest massive star-forming regions, second only to Orion KL. Cep A HW2 is the 
most studied object of all the 16 radio continuum sources reported by Hughes \& Wouterloot (1984). It is possibly the most evolved of all the objects in the region, harboring a massive star exhibiting a YSO-disk-jet system features, with the presence of a strong magnetic field and a wide-angle outflow traced by $\mathrm{H}_{2} \mathrm{O}$ masers (see reports by Rodríguez et al. 1994; Patel et al. 2005; Curiel et al. 2006; Jiménez-Serra et al. 2007; Torrelles et al. 2007, 2011; Vlemmings et al. 2010).

In this proceedings, we direct our attention to the HW3d object, located $\sim 3$ " south-east of HW2. Judging from the positive spectral index of $\mathrm{HW} 3 \mathrm{~d}$, the presence of $\mathrm{H}_{2} \mathrm{O}$ masers and OH masers (Hughes et al. 1995; Cohen et al. 1984), Garay et al. (1996) suggested that HW3d may be harboring a YSO rather than being excited externally by shocks.

\section{VERA \& VLA Observations of Cepheus A}

From 2006 May 13 to 2007 August 31, we conducted 9-epoch spectral observations toward Cep $\mathrm{A} \mathrm{H}_{2} \mathrm{O}$ masers at $\sim 22 \mathrm{GHz}$ using the VLBI Exploration of Radio Astrometry (VERA). J2005+7752, BL Lac, J2015+3710 were also observed for the calibration of the $\mathrm{H}_{2} \mathrm{O}$ maser data. Using the dual-beam system of VERA, we were able to simultaneously observe a position reference source, J2303+6405, $2.19^{\circ}$ away from Cep A for astrometric purposes. In the AIPS data reduction, we executed the self-calibration procedures using a bright maser feature in order to detect weak maser emission. We also conducted an inverse phase referencing procedure to detect the weak continuum emission from the position reference source, after which astrometry was possible in order to determine the absolute positions of the $\mathrm{H}_{2} \mathrm{O}$ masers features (see Chibueze et al. 2012). From our detected maser features in HW3d, we traced 30 relative proper motions associated with the object.

To explore possible variation in the HW3d continuum emission, we retrieved Very Large Array (VLA) $1.3 \mathrm{~cm}$ archival data of 1995 and 2006. Standard calibration procedures were applied in reducing the data. A close look at the two obtained $1.3 \mathrm{~cm}$ continuum emission maps shows some interesting variabilities (see Figure 1).

\section{Results}

\section{VLA Results}

Figure 1 shows the superimposed maps of the 1995 (red contours) and the 2006 (white contours) VLA $1.3 \mathrm{~cm}$ continuum emissions from the HW2, HW3d and HW3c. The insert therein shows the 2006 continuum while the crosses represent the positions of the continuum peaks in 1995. A couple of variations can be clearly seen in the HW3d object. First, the 1995 HW3d compact structure has evolved into an elongated structure. Second, there is a shift of $\sim 200$ mas in the position of the HW3d continuum peak between the epochs. Assuming that there is only one YSO in HW3d, we estimated its proper motion to be $\sim 65 \mathrm{~km} \mathrm{~s}^{-1}$ using the 200 mas change is its continuum peak position. We also found a variation in the total flux density of HW3d ( $~ 9$ mJy in 1995, and $~ 6.9$ mJy in 2006).

To explain the observed changes in HW3d, one may think of three possible scenarios: 1. A single YSO having a proper motion of $\sim 65 \mathrm{~km} \mathrm{~s}^{-1}$ (This is likely the most improbable considering the high velocity of the object);

2. The presence of multiple sources in HW3d;

3. Internal proper motions of clumps in a jet with flux density variation.

VERA Results

From the detected maser features in HW3d we measured, for the first time, $30 \mathrm{H}_{2} \mathrm{O}$ maser relative proper motions, by adopting a reference position derived using the method 


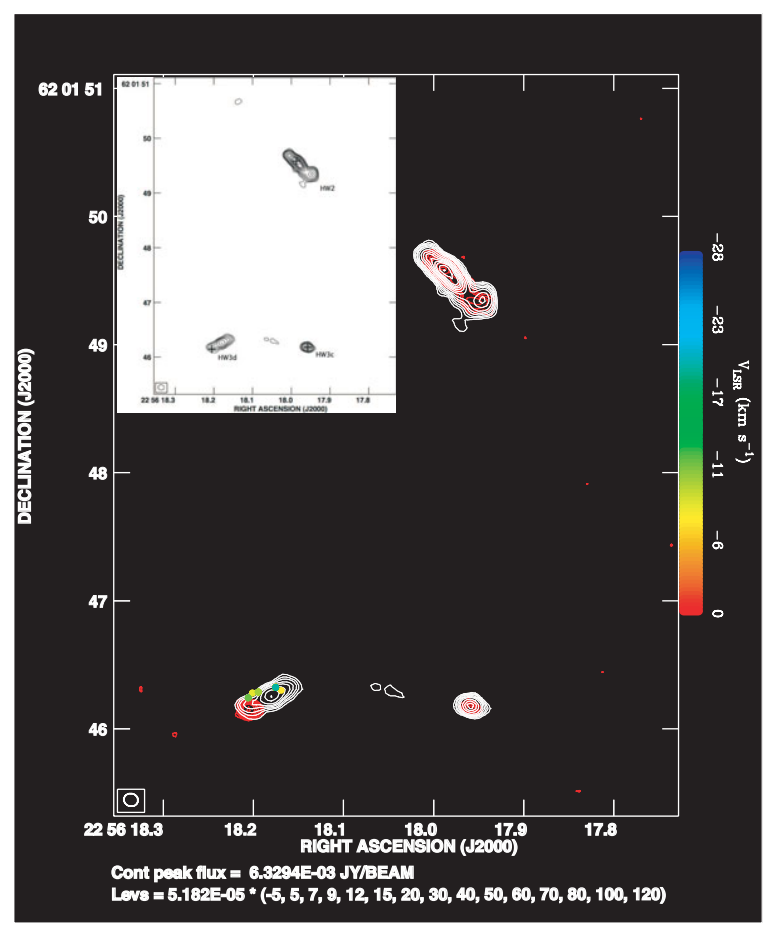

Figure 1. VLA $1.3 \mathrm{~cm}$ continuum emission map of Cep A with the $\mathrm{H}_{2} \mathrm{O}$ masers of HW3d. The white and red contours represent the 2006 and 1995 continuum respectively. Insert: 2006 map showing a cross indicating the position of the HW3d continuum peak in 1995. HW2, HW3d and HW3c are labeled appropriately (Figure adapted from Chibueze et al. 2012).

used in Torrelles et al. (2001). The proper motions show a 400-mas linear structure which is aligned with the direction of elongation of the VLA $20061.3 \mathrm{~cm}$ continuum emission. It is also bipolar in nature, thus implying that the $\mathrm{H}_{2} \mathrm{O}$ maser relative proper motions are tracing a bipolar outflow in HW3d (see Figure 2). The opening angle of the outflow derived from the deconvolved image of the continuum emission is $\sim 30^{\circ}$, showing the bipolar outflow to be highly collimated. The C-cluster of $\mathrm{H}_{2} \mathrm{O}$ maser proper motions showed high velocity dispersion $\left(\sim 20 \mathrm{~km} \mathrm{~s}^{-1}\right)$, which is suggestive of the fact that the outflow exciting source may be located in or near it (see Figure 2).

\section{Analyses and Discussion}

Position \& Velocity Variance/Covariance Matrix Analyses (PVCM \& VVCM) $\mathrm{PVCM} / \mathrm{VVCM}$, first introduced by Bloemhof (1993), is good tool for extracting the spatial and kinematic essentials from the maser positions and proper motions. It involves the diagonalization of the matrices built from the maser position and velocity (proper motion) information using

$$
\sigma_{i j}=\frac{1}{N-1} \sum_{n=1}^{N}\left(v_{i, n}-\bar{v}_{i}\right)\left(v_{j, n}-\bar{v}_{j}\right),
$$

where $i$ and $j$ denote the two dimensional space axes in the case of position, or three orthogonal space axes in the case of velocity. $n$ is the $n$-th maser feature in the collection summing up to $N(=30)$. The bar indicates averaging over the maser features. 


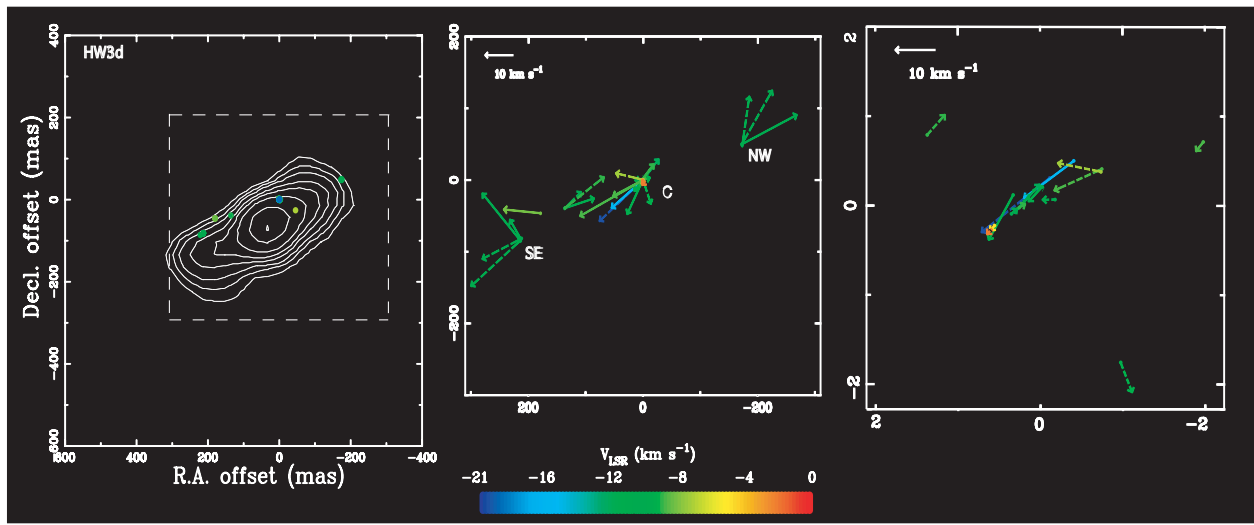

Figure 2. left: Distribution of the $\mathrm{H}_{2} \mathrm{O}$ masers detected in our VERA observations superposed on the HW3d $1.3 \mathrm{~cm}$ continuum map obtained with the VLA in 2006. middle: The proper motions of these maser features. The position, length, and direction of an arrow indicate the position, speed, and direction of the maser feature motion on the sky, respectively. The continuous lines represent proper motions traced in 3 or more epochs while the dashed lines represent those traced in 2 epochs only. The color codes represent the maser LSR velocities. right: Zoom of the C-cluster showing high velocity dispersion within a $\sim 4$ mas area (Figure adapted from Chibueze et al. 2012).

In the 2-D PVCM, the maximum eigenvalue, $\left(\psi_{\max }=12697.3 \mathrm{mas}^{2} ;\right.$ at position angle, $\mathrm{PA}, 108.5^{\circ}$ ) is $\sim 400$ times the minimum one, indicating high collimation in the maser position dispersion. In the case of the $3-\mathrm{D}$ VVCM, $\psi_{\max }\left(\mathrm{PA} 123^{\circ}\right), \psi_{\text {mid }}$, and $\psi_{\text {min }}$ are $111.4,34.9$. and $11.6 \mathrm{~km}^{2} \mathrm{~s}^{-2}$. The largest eigenvalue is 3 times the second largest eigenvalue, which shows a collimation factor of 3 in the velocity dispersion. There is correspondence between the PA of $\psi_{\max }$ in both PVCM and VVCM.

Modeling the $\mathrm{H}_{2} \mathrm{O}$ Maser Spatio-kinematics

We have also made an expanding flow model of the $\mathrm{H}_{2} \mathrm{O}$ maser proper motions based on a least-squares method (see Imai et al. 2011; Chibueze et al. 2012). We have assumed a single source scenario, located near the turbulent C-cluster, and that all the masers are moving away from the exciting source position with expansion velocities, $V_{\exp }(\mathrm{i})$ (expected to be positive). Figure 3 shows the plot of the obtained expansion velocities

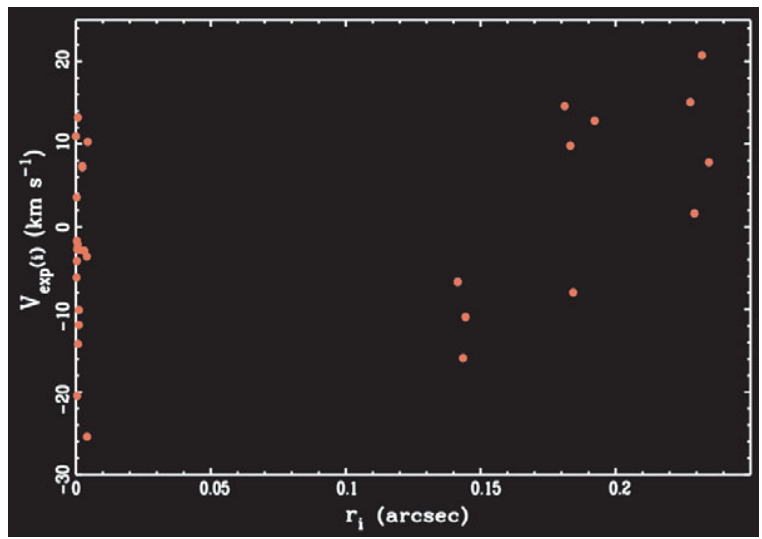

Figure 3. Expansion velocity distribution derived from the expansion flow model fitting of the maser proper motions in HW3d (Figure adapted from Chibueze et al. 2012). 
versus the offset positions from the possible position of the exciting source. Evidently, the northwest and southeast (NW, SE) clusters fit properly into the model, but the proper motions of the cluster located around (+135 mas, - 39 mas) did not fit into the expansion flow model. Near the originating center (20 mas, -17 mas), there are also proper motions with negative $V_{\exp }(\mathrm{i})$ which may be indication of infall, but the high infall velocities seen in the result may be due to another object in close proximity.

\section{Summary/Conclusion}

Based on our results and analyses we conclude the following:

1. We detected outflow activity in HW3d, indicating that this source is internally excited by a young massive stellar object.

2. The bipolar motions of the $\mathrm{H}_{2} \mathrm{O}$ masers indicate the presence of a YSO-Jet system in HW3d.

3. The shift in position and flux variation of the continuum emission in HW3d suggests the possibility of multiplicity in the region or internal proper motions of clumps in the jet.

4. This multiplicity and/or proper motions of clumps in the jet can be tested in the future with SMA and EVLA observations.

\section{References}

Bloemhof, E. E. 1993, ApJ, 406, L75

Bonnell, I. A., Bate, M. R., \& Zinnecker, H., 1998, MNRAS, 298, 93

Bonnell, I. A. \& Bate, M. R., 2006, MNRAS, 370, 488

Chibueze, J. O., Imai, H., Tafoya, D., et al. 2012, ApJ, in press

Cohen, R. J., Rowland, P. R., \& Blair, M. M., 1984, MNRAS, 210, 425

Curiel, S., Ho, P. T. P., Patel, N. A., et al. 2006, ApJ, 638, 878

Dzib, S., Loinard, L., Rodríguez, L. F., Mioduszewski, A. J., \& Torres, R. M., 2011, ApJ, 733,71

Garay, G., Ramírez, S., Rodríguez, L. F., Curiel, S., \& Torrelles, J. M., 1996, ApJ, 459,193

Hughes, V. A. \& Wouterloot, J. G. A., 1984, ApJ, 276, 204

Hughes, V. A., Cohen, R. J., \& Garrington, S., 1995, MNRAS, 272, 469

Imai, H., Omi, R., Kurayama, T., et al. 2011, PASJ, 63, 1293

Jiménez-Serra, I., Martín-Pintado, J., Rodríguez-Franco, A., et al. 2007, ApJ, 661, L187

Patel, N. A., Curiel, S., Sridharan, et al. 2005, Nature, 437, 109

Rodríguez, L. F., Garay, G., Curiel, S., et al. 1994, ApJ, 430, L65

Torrelles, J. M., Patel, N. A., Gómez, J. F., et al. 2001b, ApJ, 560, 853

Torrelles, J. M., Patel, N. A., Curiel, S., et al. 2007, ApJ, 666, L37

Torrelles, J. M., Patel, N. A., Curiel, S., et al. 2011, MNRAS, 410, 627

Vlemmings, W. H., T., Surcis, G., Torstensson, K. J. E., \& van Langevelde, H., J., 2010, MNRAS, 404,134

Yorke, H. W. \& Sonnhalter, C., 2002, ApJ, 569, 846

Zinnecker, H. \& Yorke, H. W., 2007, ARA\&A, 45, 881 